\title{
A Sensor Awakening Algorithm for Wireless Multimedia Sensor Networks Three Dimensional Target Tracking
}

\author{
Jing Zhao ${ }^{1,2}$, Jianchao Zeng ${ }^{2}$ \\ ${ }^{1}$ College of Electrical and Information Engineering, Lanzhou University of Technology, Lanzhou, China \\ ${ }^{2}$ Complex System and Computational Intelligence Laboratory, Taiyuan University of Science and Technology, \\ Taiyuan, China \\ E-mail: zhaojing_740609@163.com, zengjianchao@263.com \\ Received May 13, 2010; revised June 22, 2010; accepted July 24, 2010
}

\begin{abstract}
For node awakening in wireless multi-sensor networks, an algorithm is put forward for three dimensional target tracking. To monitor target dynamically in three dimensional area by controlling nodes, we constract virtual force between moving target and the current sense node depending on the virtual potential method, then select the next sense node with information gain function, so that when target randomly move in the specific three dimensional area, the maximum sensing ratio of motion trajectory is get with few nodes. The proposed algorithm is verified from the simulations.
\end{abstract}

Keywords: Wireless Multimedia Sensor Network, Multimedia Sensor, Sense Area, Possible Sense Area, Three Dimensional Target Tracking, Information Gain, Virtual Potential

\section{Introduction}

Wireless sensor networks (WSNs) have drawn a more attention in the last few years [1], including traditional wireless sensor networks and wireless multimedia sensor networks(WMSNs). Since the traditional wireless sensor networks only provide simple sensing data such as temperature, humidity and so on [2], so not as to meet the requirement of more complicated and precise data applications. WMSNs differ from the traditional wireless sensor networks due to their characteristic of directivity and are more interest in intensive information data (e.g. video, image) [3].

The theory of the virtual force is often used to solve coverage problem in WSNs. It was first proposed in the research of the mobile robotics route plan and obstacle avoidance by Khatib [4]. Howard et al [5] applied it to the coverage problem of WSNs, then the technique was proved to be useful for such problem in [6,7], the virtual potential field could cause the repel force between sensors. The force that repelled each other made sensor spread from dense to sparse area. Tao et al. and Zhao et al. $[8,9]$ present a virtual potential field based coverage-enhancing algorithm for directional sensor networks, where overlap is reduced and the ratio of coverage is enhanced by forcing sensors to the most beneficial orientation under repel force in terms of the Euclidean distance be- tween 'centroid'.

There are many target tracking algorithms for traditional wireless sensor networks, such as following[10]: K Mechitov et al [11] provide an cooperative tracking with binary-detection algorithm; In [12], based on signal intensity a distributed algorithm about decentralized source localization and tracking is put forward by Rabbat and Nowak; Based on clusering, Friedlander D. et al. provides Dynamic space-time algorithms[13]; A Adaptive target tracking algorithms is given by Xingbo Yu et al. by considering tracking efficiency and nodes energy consumption[14]; Gordon [15] uses particle filter algorithm and so on, but most of them focus on the research of target location and data processing. The coverage is a fundamental problem in the networks [16]. Perfected coverage is important to sense target area and collect useful data. After node freedom deployment, it is a hot problem needed to be urgently solved that how control sensors to cover motion trajectory efficiently and wholly [17] especially for three dimensional target tracking.

In this paper, focus on three dimensional target tracking application, we structure virtual force between moving target and the current sense node based on the virtual potential method, and control node rotation to get maximum sensing ratio of motion trajectory with few sensors, and define the warning round, intersection and information gain area, then select the next sense node with in- 
formation gain function so that as few nodes as possible are used to get the maximum probability of motion trajectory sensing when target randomly move in the specific three dimensional area.

\section{Sensing Model}

\subsection{Concepts}

Prior to build sensing model of WMSNs, some definitions are given below:

- Sense Cone.

Sensing cone is the three dimensional area being sensed by multimedia sensor in three dimensional target area, shown with shadow area in Figure 1.

- Possible Sense Area.

Possible sense area is a sphere being sensed by multimedia sensor rotation in three dimensional target area, shown with all sphere in Figure 2.

- Sense Direction.

In three dimensional target area, three dimensional coordinate axis is built through sensor position, sense direction, that is $\vec{\alpha}$, are expressed by $(\beta, \theta, \omega)$ those respectively are vidicon's offset angles to the X, Y AND $\mathrm{Z}$ coordinate planes.

- Neighboring Sensor.

The neighboring sensors are the sensors whose distances are less than $2 \mathrm{R}$, where the $\mathrm{R}$ is the radius of Possible Sense Area.

- Neighboring Area and Neighboring Sensor of Intersection.

The neighboring area of intersection is the circle area whose radius to intersection is less than $\mathrm{R}$ and the sensors in this circle are neighboring sensor of intersection.

- Warning Round.

The warning round is the circle whose radius is less than $\mathrm{R}$, when target arrive at warning circle, sense node

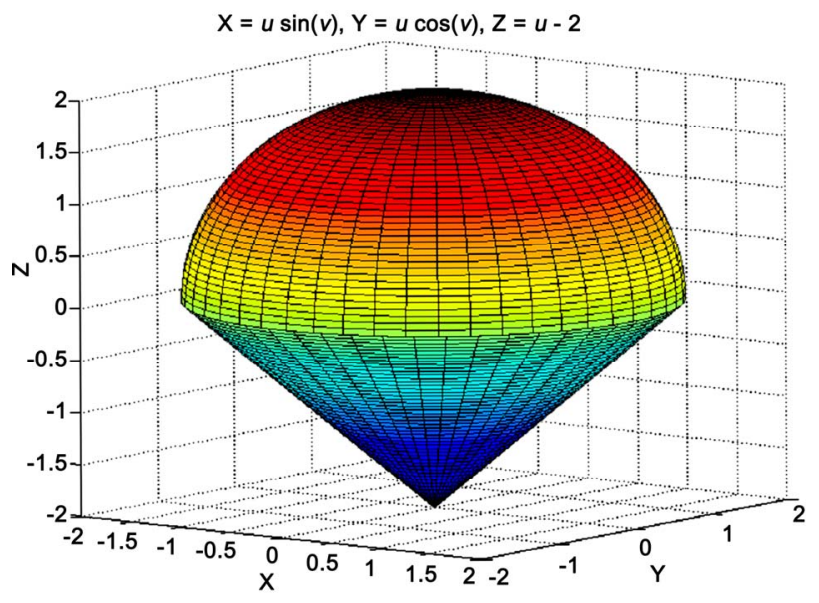

Figure 1. Sensing cone of a sensor.

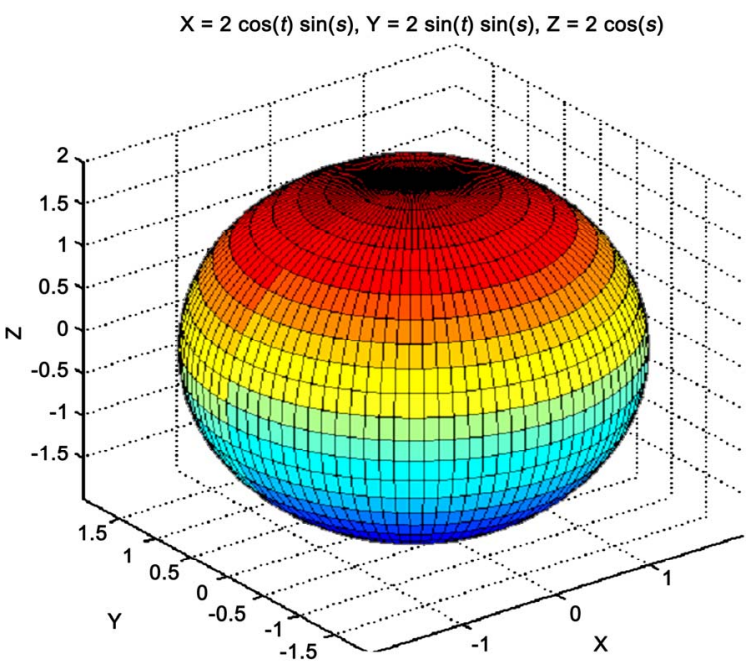

Figure 2. Possible sensing area of a sensor.

will warn other neighboring sensors of this intersection and the next sense node begin to be selected.

- Intersection.

The intersection is the join point of warning round and target motion trajectory and target will continue moving with direction at this point.

- Centroid.

The "centroid" point denotes center of the mass in physics. Here, the "centroid" is defined as the center of sense cone, so the sector's turning around the location is viewed as the centroid's rotation. The "centroid" point of sense cone is at the symmetric axis and its distance to sensor location is $2 R \sin (\alpha) / 3 \alpha$.

- Coverage Leak.

The coverage leak is the uncovered motion trajectory of target.

- Coverage Ratio of Target.

That is ratio of motion trajectory length covered by all sense nodes' possible sense area to whole motion trajectory length.

\subsection{Sensing Models}

Unlike an isotropic sensor, a multimedia sensor has a cone sensing area shown in Figure 1 and aimed at maximizing covered motion trajectory with a minimum number of sensors, it is assumed not to move and only to rotate around sensor position after randomly deployed, so forms sphere possible sensing area, as shown in Figure 2.

Considering sense cone and possible sense sphere,we define sensing model with 5-tuple, shown as follows: $(P, R, \vec{\alpha}, \phi, v)$, there $\mathrm{P}$ is sensor's location, $\mathrm{R}$ is radius of sector'area, $\vec{\alpha}$ shows sensor's sense direction, $\phi$ shows half of separation angle of sensing cone, $v$ is rotational speed, that is sensor rotational speed for sensing moving target. 


\section{A Sensor Awakening Algorithm for Wireless Multimedia Sensor Networks Three Dimensional Target Tracking (SATTT)}

\subsection{Problem Definition}

We shall study the problem of node scheduling in wireless sensor networks. To begin with, some necessary assumptions have to be made: 1) the localization information of moving target can be achieved with certain equipments built in sensors such as radar, infrared aids, and angle measuring device; 2) the exact sensing angle and position of sensor can be gained by itself; 3) all nodes in network are homogeneous, whose sensing angle and radius are same as each other; and 4) all sensor nodes are deployed randomly.

In real monitor applications, the target randomly enter the specific three dimensional area and randomly move in this area till it leaves the area. Then the controlling of sensing nodes, i.e., awaking as less nodes as possible to monitor the target gets inevitable. Aiming at the surveillance for target randomly moving in the specific area, this paper develops a node controlling algorithm for three dimensional target sensing with randomly deployed nodes in wireless multimedia sensor network.

\subsection{Idea of Algorithm}

Entering the specific three dimensional area, the target will be sensed by the one node, so others sensors turn to be asleep. Then the virtual gravitational force between target and sensing node can be calculated based on virtual potential theory and used to control the node turned around following with the moving target. When the target moves at the warning around of the node possible sensing sphere, the intersection is formed. According to the moving angle of target at the intersection, the area of information gain is set to calculate the gain values of the neighbor nodes of the intersection. The node having the maximum gain value will be selected to be the next sense node. At the same time, the current sensing node is turned to be asleep. The above process repeats until the target goes away the specific area.

\subsection{Sensor Rotation Way}

Entering the specific three dimensional area, the target will be sensed by one nodes. Then the virtual force between target and sensing node can be calculated based on virtual potential theory and used to control the node to rotate following with the target movement.

$$
\vec{F}_{i}=k_{1} \times \frac{1}{r_{i}^{2}} \times \overrightarrow{r_{0 i}}
$$

where $k_{1}$ is density of field; $\vec{r}_{0 i}$ is a vector of unit length and describes the direction of force from "centroid" point of node $i$ to target location; $r_{i}$ describes distance between "centroid" point of node $i$ and target location. When target is sensed by sensor $i$, sensor $i$ will rotate following with target moving under virtual force coming from target, so sensor rotational speed is decided by the target moving speed.

\subsection{Selection of Sensor Node}

When target moves at the boundary of the sense node, selecting the next effective sense node is very important to target tracking.

- Formation of Intersection.

When the target moves at the warning round of the sense node, the intersection point of warning round and motion trajectory forms the intersection of sense node, as shown in Figure 3. Where, the bigger sphere shown with mesh grid describes possible sense round of sensor $i$, the smaller solid sphere is warning round, black line MN describes target motion trajectory, $\mathrm{A}$ is the intersection of sense node $i$.

- Setting area of Information Gain.

In this paper, information is the covered length of motion trajectory that is uncovered before, gain is increasing and describes information increasing, so the area of information gain is the area where uncovered motion trajectory is covered by next sense node, that is explained in Figure 4, where MA is the target moving direction at

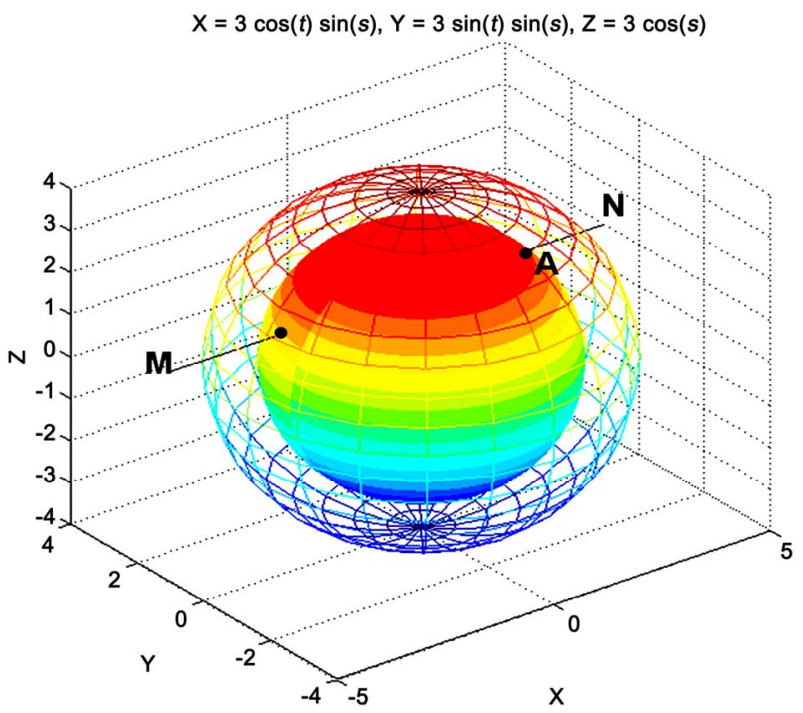

Figure 3. Intersection of sense node. 


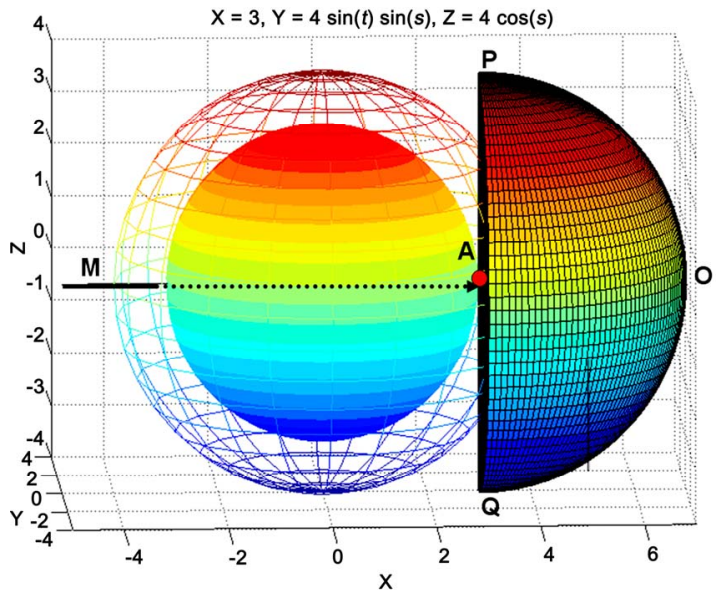

Figure 4. Information gain area.

intersection $\mathrm{A}, \mathrm{PQ}$ is vertical plane of $\mathrm{MA}$ and through intersection $\mathrm{A}$, neighboring area of intersection $\mathrm{A}$ is divided equally, the half sphere POQ pointed at by MA is the area of information gain. The area is a estimation area where the sensor the most likely covers coming motion trajectory.

- Calculation of Information Gain and Selection of Next Sense Node.

In real monitor applications, the scheduling of sensing nodes, i.e., awaking as less nodes as possible to monitor the target gets inevitable, so for getting the maximum information gain, the next sense node is so far so good from point $\mathrm{A}$ and the distance from the next sense node to Linear $\mathrm{AE}$ is as close as possible, that is explained with ichnography in Figure 5, where $\mathrm{X}$ is random sensor in information gain half sphere, XY describes the distance from $\mathrm{X}$ to Linear $\mathrm{AE}, \mathrm{AX}$ is the distance from $\mathrm{X}$ to intersection $\mathrm{A}$, information gain is determined by $\mathrm{AX}$ and $\mathrm{XY}$, shown as follows:

$$
\text { inf }=k_{2} \times \frac{\mathrm{AX}}{\mathrm{XY}}
$$

\section{Simulation Results}

The Simulation Results of controlling algorithm and the effect of sensor parameters on coverage ratio is given in this section.

\subsection{Simulation Results of the SATTT}

To simulate the algorithm, 20 isomorphic sensors with sensing radius $60 \mathrm{~m}$ and separation angle $45^{\circ}$ of sensing cone are deployed randomly in a region of $500 \times 500$ $\times 500 \mathrm{~m}^{3}$. Entering this specific three dimensional area randomly, the target will move randomly till living it. The simulation results are shown in Figures 6-7, where,
Figure 6 shows the initial sensors deployment in the region and little spheres show location of sensors; Fig.7 shows the coverage ratio of target motion trajectory, where the target enter the area from A and leaves from B after randomly moving in the three dimensional area, and spheres show awaked sensors for sensing target, the red line with stare dots is target motion trajectory whose coverage ratio is $32.26 \%$.

When 200 isomorphic sensors are deployed randomly, the simulation results are shown in Figures 8-9, coverage ratio is $100 \%$.

\subsection{Effects of Sensor Number on Coverage Ratio}

To simulate the effect of sensor number on coverage ratio, sensors with sensing radius $60 \mathrm{~m}$ and separation angle $45^{\circ}$ of sensing sector are deployed randomly in a

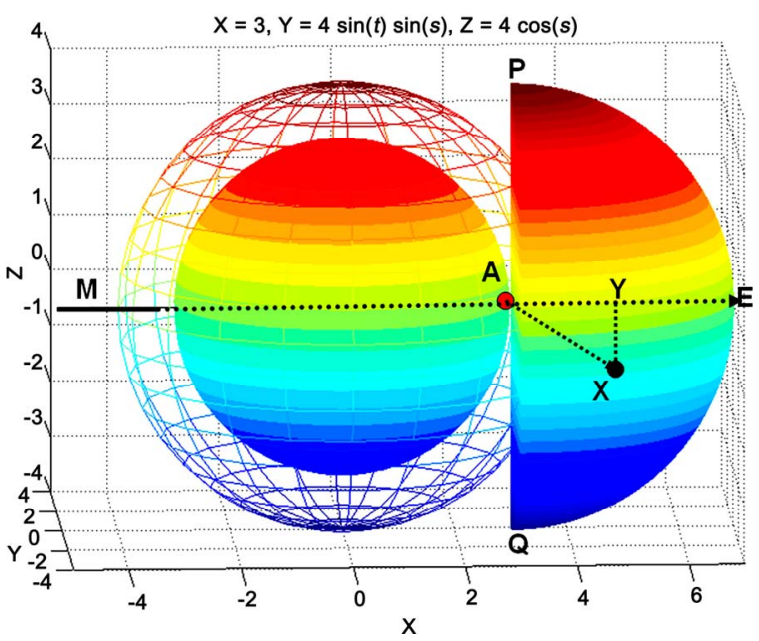

Figure 5. Forecast of sense node.

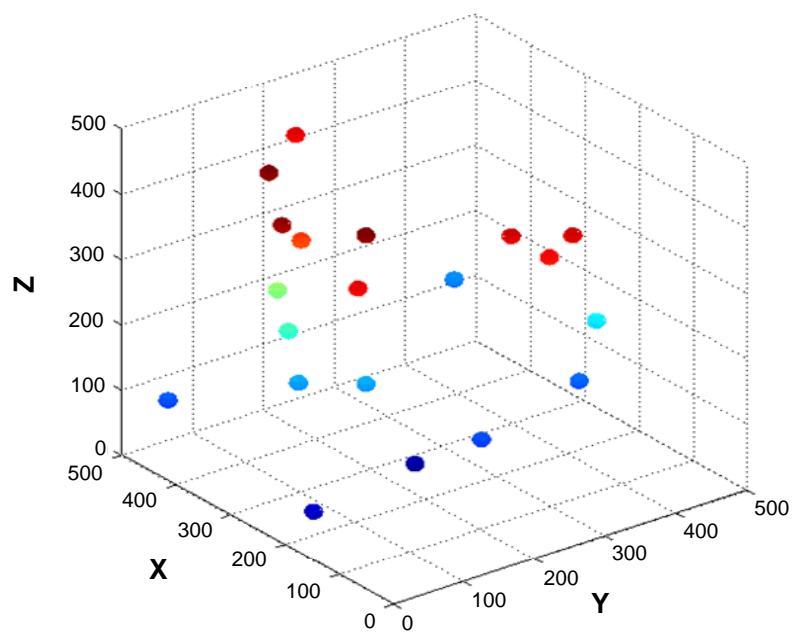

Figure 6. Initial deployment of 20 sensors. 


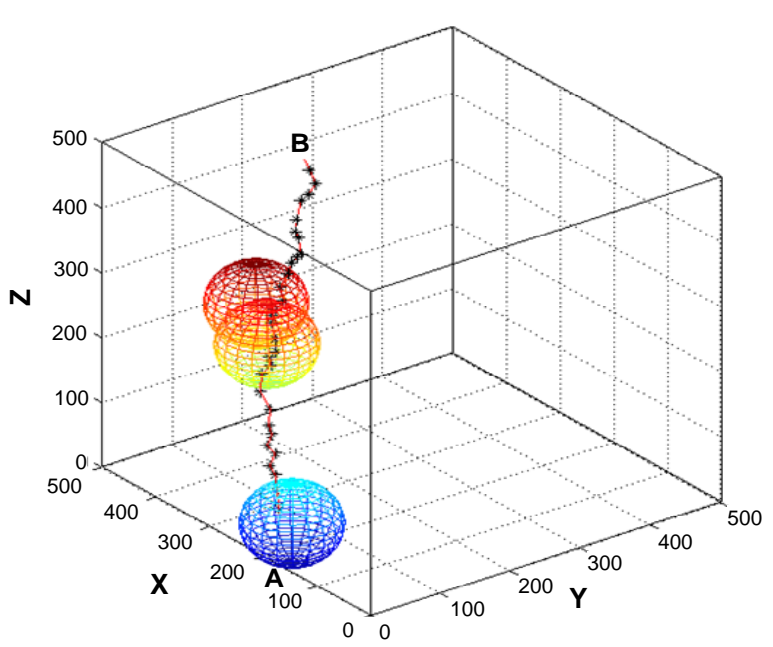

Figure 7. Node scheduling of 20 sensors, $p=32.26 \backslash \%$.

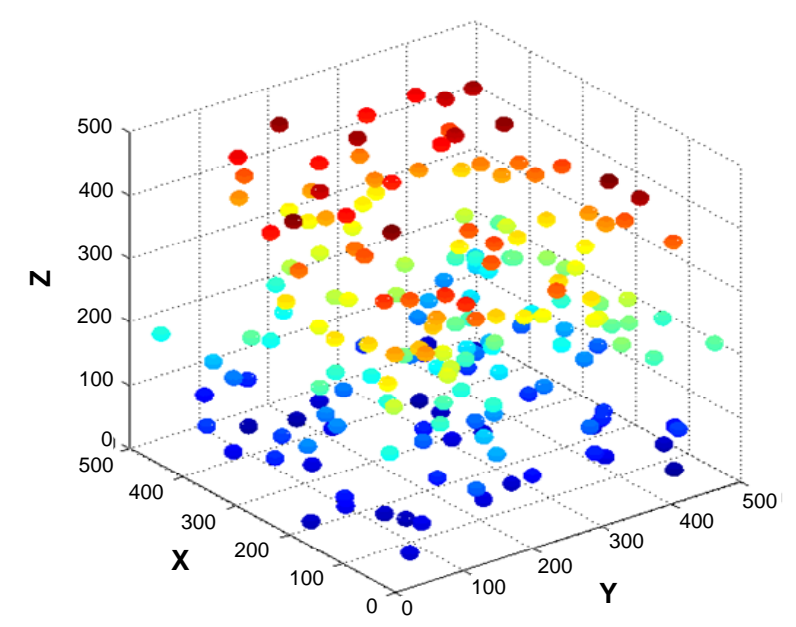

Figure 8. Initial deployment of 200 sensors.

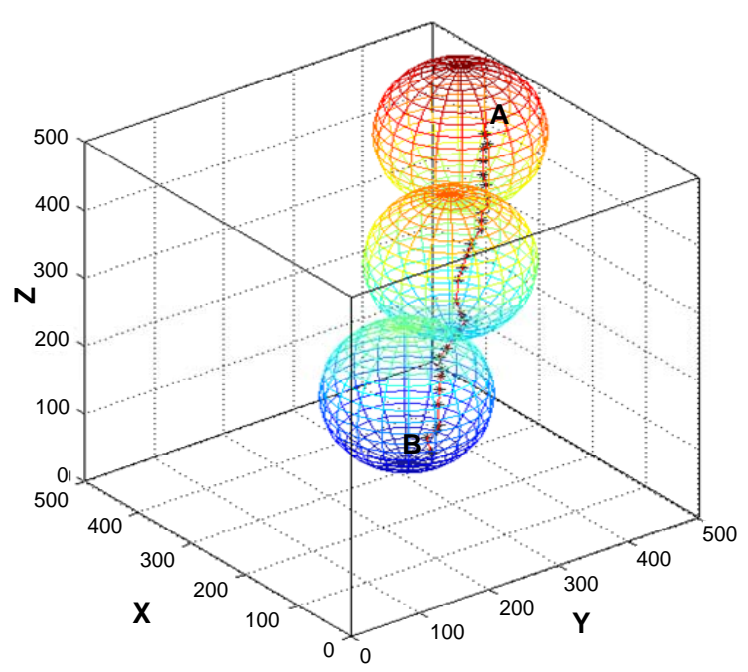

Figure 9. Node scheduling of 200 sensors, $p=100 \backslash \%$. region of $500 \times 500 \times 500 \mathrm{~m}^{3}$. We provide the mean results of many simulations with 50, 100, 150 and 200 sensors in Figure 10. It shows that as the number of nodes increases, the coverage ratio of motion trajectory increase too.

\subsection{Effects of Sense Radius on Coverage Ratios}

To simulate the effect of sense radius on coverage ratios, 200 sensors with separation angle $45^{\circ}$ of sensing sector are deployed randomly in a region of $500 \times 500 \times 500 \mathrm{~m}^{3}$. We provide the mean results of simulations with sensing radius $60 \mathrm{~m}, 80 \mathrm{~m}$ and $100 \mathrm{~m}$ in Figure 11. It shows that the coverage ratio of motion trajectory increases following with increasing of nodes sensing radius and nearly reaches $p=100 \%$ when radius is 100 .

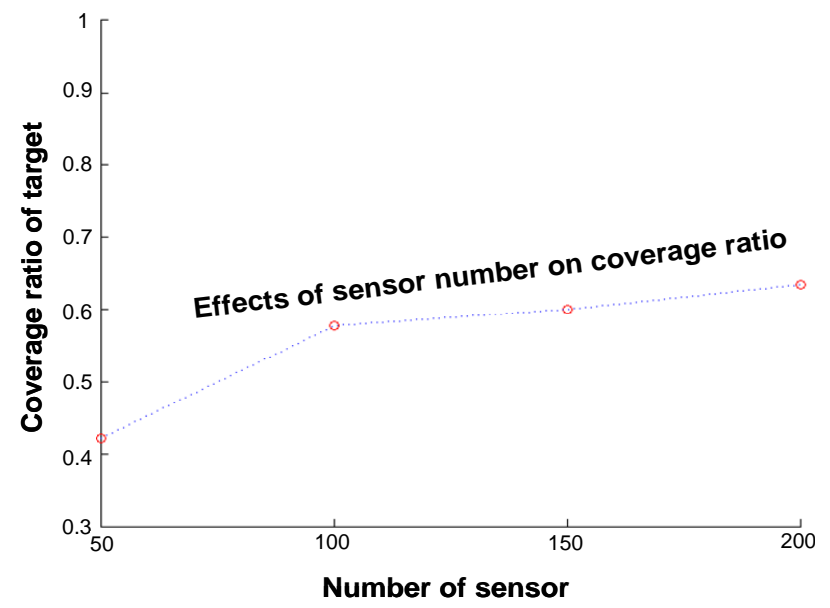

Figure 10. Effects of sensor number on coverage ratio.

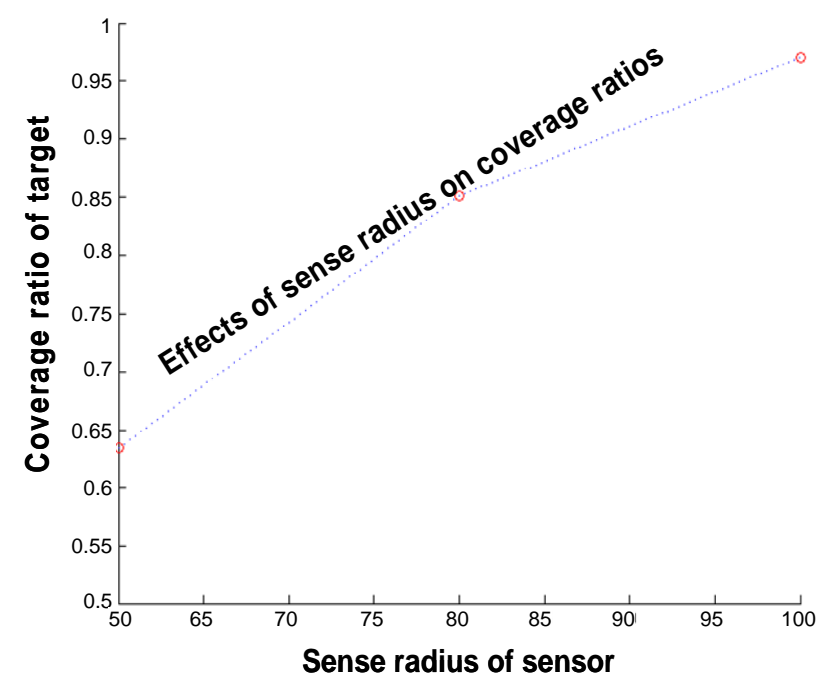

Figure 11. Effects of sense radius on coverage ratio. 


\section{Conclusions}

Concentrating on node controlling, the authors put forward an algorithm for three dimensional target tracking in wireless multi-sensor networks. The corresponding algorithm working depends on the virtual potential method. To monitor target dynamically by controlling nodes, we structure virtual force between moving target and the current sense node, then select the next sense node with information gain function so that as few nodes as possible are used to get the maximum probability of motion trajectory sensing when target randomly move in the specific three dimensional area till target living it. The proposed algorithm is verified from the simulations.

\section{References}

[1] I. F. Akyildiz, T. Melodia and K. R. Chowdhury, "A Survey on Wireless Multimedia Sensor Networks," Computer Networks, Vol. 51, No. 4, March 2007, pp. 921960.

[2] I. Akyildiz, W. Su, Y. Sankarasubramaniam and E. Cayirci, "Wireless Sensor Networks: A Survey," Computer Networks, Vol. 38, No. 4, 2002, pp. 393-422.

[3] H. Ma and D. Tao, "Multimedia Sensor Network and its Research Progresses," Journal of Software, Vol. 17, No. 9, 2006, pp. 2013-2028.

[4] O. Khatib, "Real-Time Obstacle Avoidance for Manipulators and Mobile Robots," The International Journal of Robotics Research, Vol. 5, No. 1, 1986, pp. 90-98.

[5] A. Howard, M. Mataric and G. Sukhatme, "Mobile Sensor Network Deployment Using Potential Fields: A Distributed, Scalable Solution to the Areacoverage Problem," Proceedings of 6th International Symposium on Distributed Autonomous Robotic Systems, Fukuoka, Vol. 5, 2527 June 2002, pp. 299-308.

[6] X. Wang, S. Wang and J. Ma, "An Improved Co-Evolutionary Particle Swarm Optimization for Wireless Sensor Networks with Dynamic Deployment," Sensors, Vol. 7, No. 3, 2007, pp. 354-370.

[7] Y. Zou and K. Chakrabarty, "Sensor Deployment and Target Localization Based on Virtual Forces," Proceedings of 22nd Annual Joint Conference of the IEEE
Computer and Communications Societies, San Francisco, Vol. 2, 30 March-3 April 2003, pp. 1293-1303.

[8] D. Tao, H. Ma and L. Liu, "A Virtual Potential Field Based Coverage-Enhancing Algorithm for Directional Sensor Networks," Journal of Software, Vol. 18, No. 5, 2007, pp. 1152-1163.

[9] J. Zhao and J.-C. Zeng, "A Virtual Potential Field Based Coverage Algorithm for Directional Networks," Chinese Control and Decision Conference, Guilin, 17-19 June 2009, pp. 4605-4610.

[10] J. Tang, H.-S. Shi and Z.-X. Han, "An Target Tracking Algorithm for Wireless Sensor Networks," Journal of Air Force Engneering University (Natural Science Edition), Vol. 7, No. 5, October 2006, pp. 25-29.

[11] K. Mechitov, S. Sundresh, Y. Kwon, et al., "Agha Cooperative Tarcking with Binary-Detection Sensor Networks," Proceedings of the 1st Intemational Conference on Embedded Networked Sensor Systems, Los Angeles, 5-7 November 2003, pp. 332-333.

[12] M. G. Rabbat and R. D. Nowak, "Decentralized Source Localization and Tracking," Proceedings of the IEEE Intemational Conference on Acoustics, Speech and Signal Processing, Montreal, 17-21 May 2004, pp. 921-924.

[13] D. Friedlander, C. Griffin, N. Jacobson, et al., "Dynamic Agent Classification and Tracking Using an Ad Hoc Mobile Acoustic Sensor Network," EURASIP Joumal on Applied Signal Processing, Vol. 2003, No. 4, 2003, pp. 371-377.

[14] X. B. Yu, K. Niyogi, S. Mehrotra and N. Venkatasubramanian, "Adaptive Target Tracking in Sensor Networks," Communication Networks and Distributed Systems Modeling and Simulation Conference, San Diego, 18-21 Januray 2004, pp. 253-258.

[15] N. J. Gordon, D. J. Salmond and A. F. M. Smith, "Novel Approach to Nonlinear/Non-Gaussian Bayesian State Estimation," IEE Proceedings of Radar and Signal Processing, Vol. 140, No. 2, 1993, pp. 107-113.

[16] J. Ai and A. Abouzeid, "Coverage by Directional Sensors in Randomly Deployed Wireless Sensor Networks," Journal of Combinatorial Optimization, Vol. 11, No. 1, 2006, pp. 21-41.

[17] D. Tao, H. Ma and L. Liu, "Study on Path Coverage Enhancement Algorithm for Video Sensor Networks," Acta Electronica Sinica, Vol. 36, No. 70, 2008, pp. 1291-1296. 